\title{
Postoperative Acute Submandibular Sialadenitis after Neurosurgery: Two Case Reports and a Review of the Literature
}

\author{
Haruto Uchino, Hiroaki Motegi, Hiroyuki Kobayashi, Yuuta Kamoshima, Ken Kazumata, \\ Shunsuke Terasaka, and Kiyohiro Houkin
}

\begin{abstract}
This article details two case reports and reviews the extant literature concerning acute postoperative submandibular sialadenitis occurring in the side contralateral to the operated site after neurosurgery. Although its precise pathogenesis remains to be elucidated, it is likely related to intraoperative compression of the submandibular gland and surrounding tissues, caused by head positioning and the endotracheal tube. Submandibular swelling rapidly deteriorates after surgery, and emergent airway protection is required in most cases in order to avoid fatal airway obstruction. To avoid serious sequelae, we should be aware of acute submandibular sialadenitis that occurs contralateral to the surgical side, which, although rare, is more probable after posterior fossa surgery. When it occurs, early airway protection is crucial, and the following conservative treatment could provide a good prognosis.
\end{abstract}

Keywords: neck swelling, posterior fossa surgery, respiratory distress, submandibular sialadenitis, surgical complication

\section{Introduction}

In rare instances, patients develop acute submandibular sialadenitis after a neurosurgical operation, which results in impending airway obstruction. This occurs on the side opposite to the surgery site and is likely related to head rotation and/or flexion during surgery. Although such situations are unique, emergent airway protection is usually required when cases do occur. We present two cases of acute submandibular sialadenitis contralateral to the operated side after posterior fossa craniotomy. We also consider its pathogenic mechanism, with an emphasis on anatomical structures. Finally, we briefly review the extant literature on the subject.

\section{Case Reports}

\section{Case 1}

A 20-year-old healthy man (height, $173 \mathrm{~cm}$; weight, $55 \mathrm{~kg}$ ) diagnosed with vestibular schwannoma underwent a right retrosigmoid suboccipital craniotomy. After induction of anesthesia, a 7.5-mm endotracheal intubation was performed. The endotracheal tube was fixed on the left corner of the

Department of Neurosurgery, Graduate School of Medicine, Hokkaido University, Sapporo, Hokkaido

Received: March 13, 2014; Accepted: July 13, 2015 mouth and electrode needles were inserted into the right facial muscles to monitor his facial electromyographic (EMG) responses (Fig. 1). He was placed in the left park bench position, where the head was fixed in a Mayfield clamp. The neck was moderately flexed, and the head was elevated to the lower shoulder and maintained a horizontal position with one vertex down. The face was rotated slightly down. A twofinger width between the chin and thorax was confirmed.

The patient's position remained stable throughout the 8 -hour procedure. The total amount of fluid infused, the urine output, and the blood loss were 4,550 mL; 3,200 mL; and $70 \mathrm{~mL}$; respectively. After surgery, he was conscious and was extubated without any problems.

The left neck swelling began 4 hours after the operation and progressed over the next 12 hours. The patient started complaining of numbness of the tongue and respiratory distress. A computed tomography (CT) scan indicated marked swelling of the submandibular gland and the surrounding soft tissue compressing the trachea, which was shifted to the right side (Fig. 2a). The patient was re-intubated under sedation

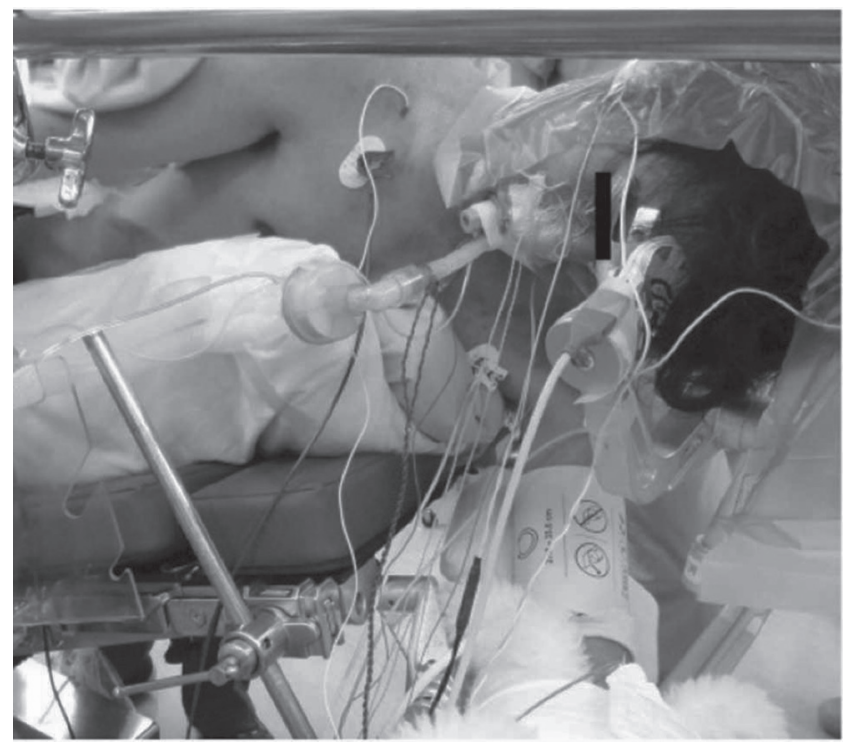

Fig. 1 Photograph of preoperative setting for tumor resection of the right side vestibular schwannoma in Case 1. An endotracheal tube was placed on the left angle of his mouth, the side contralateral to the operation. We placed electrode needles into the right (ipsilateral) facial muscles to monitor his facial electromyographic responses. 

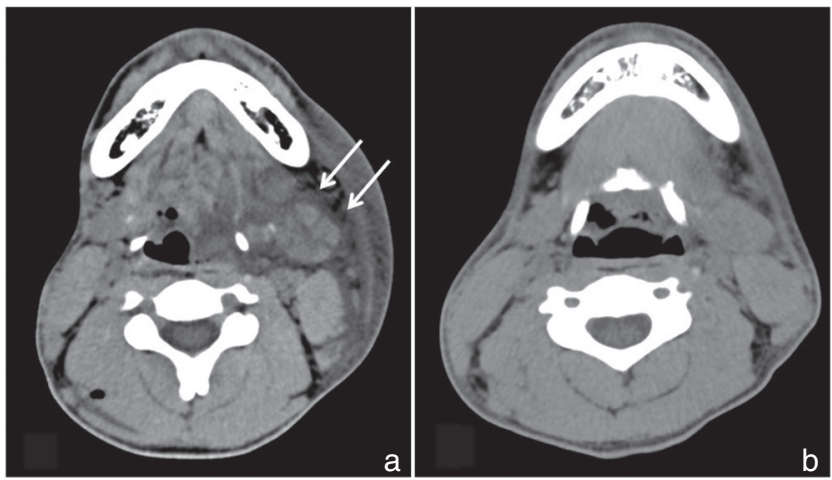

Fig. 2 a: In Case 1, computed tomography (CT) 12 h after surgery shows remarkable swelling of the left submandibular gland (arrows) and surrounding soft tissue. The larynx is compressed and deformed. b: CT 7 days after surgery demonstrates the improvement of the edematous change of the left submandibular gland and the larynx.

to avoid impending airway obstruction. No local sign of infection was present, and the patient was afebrile. Serum amylases increased slightly up to $203 \mathrm{U} / \mathrm{L}$ (normal value $<159 \mathrm{U} / \mathrm{L}$ ). After administration of antibiotics and corticosteroids, the submandibular edema regressed rapidly within 7 days (Fig. 2b), and the endotracheal tube was removed on the third treatment day. Serum amylases returned to normal range $(58 \mathrm{U} / \mathrm{L})$ on the seventh treatment day. Finally, the neck swelling completely disappeared, and the patient was discharged without any neurologic deficit on the 20th postoperative day.

\section{Case 2}

A 71-year-old woman (height, $160 \mathrm{~cm}$; weight, $53 \mathrm{~kg}$ ) presented with a 2-year history of right hemifacial spasms. A microvascular decompression was performed via right suboccipital craniotomy under general anesthesia, with a $7.5-\mathrm{mm}$ endotracheal tube. The endotracheal tube and electrode needles were fixed as in Case 1. She was placed in the left lateral position, and the head was fixed with three-point fixation to keep the nose parallel with the floor and the cranial vertex tilted slightly down towards the floor.

After a 3-hour surgery, extubation was performed in the operating room. Two hours after the operation, she developed wheezing, and an oral examination revealed an edema of the larynx without any injury. She was re-intubated and mechanically ventilated under sedation. A CT scan demonstrated an enlarged left submandibular gland and swollen surrounding soft tissues (Fig. 3). No calculus was observed in the glandular ducts. She was afebrile, and no focal sign of inflammation was apparent. Although tracheostomy was required to treat complicated aspiration pneumonia, the submandibular gland swelling and her general condition gradually improved after the administration of antibiotics and corticosteroids. The patient returned to normal life without any deficit.

\section{Discussion}

Patients sometimes complain of respiratory distress after

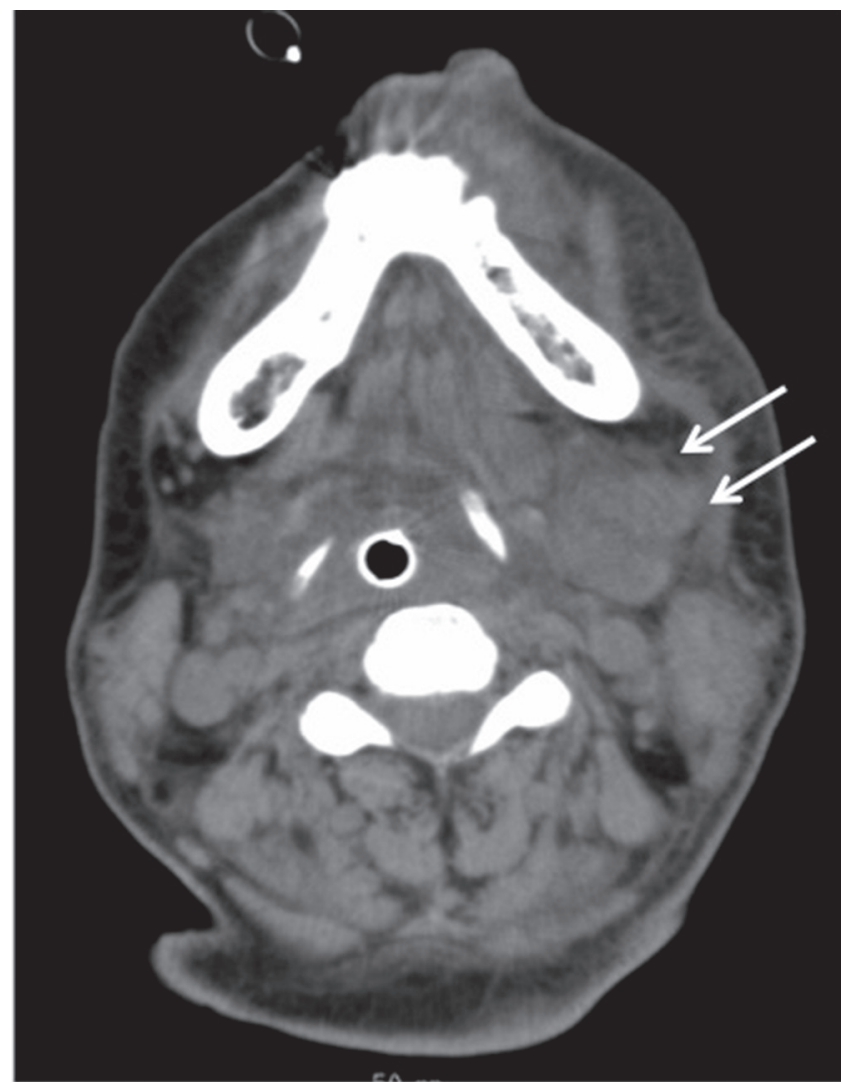

Fig. 3 In Case 2, computed tomography 2 hours after surgery shows an enlarged submandibular gland (arrows) and edematous surrounding soft tissues. The patient was re-intubated.

general anesthesia, due to minor trauma of the larynx or the vocal cord from intubation. However, unilateral acute submandibular sialadenitis after surgery is distinct and has different complication. Progression of submandibular swelling is so rapid that emergent re-intubation is required in most cases. ${ }^{1}$ The swelling of the soft tissue may sometimes cause brachial plexopathy. ${ }^{2,3)}$ Nevertheless, acute submandibular sialadenitis basically has a good prognosis with conservative treatment, if early airway protection is performed; therefore, awareness of this complication at its beginning is crucial. A CT scan or magnetic resonance imaging (MRI) is useful for the diagnosis by demonstrating an enlarged submandibular gland and an edema of the surrounding soft tissue.

Kim et al. reported this complication as being $0.84 \%$ of all retrosigmoid and far-lateral approaches. ${ }^{4)}$ From 2002 to 2012, 253 surgeries of the posterior fossa were performed by the senior author (ST), and only the two patients presented here $(0.79 \%)$ were diagnosed with postoperative acute sialadenitis.

According to the literature written in English (Table $1){ }^{1-3,5-8)}$ the affected age of patients who showed unilateral submandibular sialadenitis ranged from 20 years to 73 years old. Surgical positions adopted and the operation time also varied in these cases.

As shown in Table 1, most of the procedures were posterior fossa surgery, and all submandibular swelling occurred on 
Table 1 Summary of cases with unilateral submandibular sialadenitis after neurosurgical procedures

\begin{tabular}{|c|c|c|c|c|c|c|c|c|c|}
\hline \multirow[t]{2}{*}{ Authors } & \multirow[t]{2}{*}{ Age/Sex } & \multirow[t]{2}{*}{ Diagnosis } & \multicolumn{2}{|c|}{ Surgery } & \multicolumn{5}{|c|}{ Postoperative submandibular swelling } \\
\hline & & & Position & Duration & Onset & Side & Intubation & Treatment & Outcome \\
\hline Izci et al., 20055) & $28 / \mathrm{F}$ & Optic nerve meningioma & Supine & $5 \mathrm{~h}$ & Immediate & Contralateral & No & 7 days & Good \\
\hline Kim et al., 2008) & $\begin{array}{c}20-64 / \\
\mathrm{M}(3), \mathrm{F}(2)\end{array}$ & $\begin{array}{c}\text { meningioma (3), VS (1), } \\
\text { and cavernous } \\
\text { malformation (1) }\end{array}$ & $\begin{array}{c}\text { Supine (4) } \\
\text { Park-bench (1) }\end{array}$ & $3-6 \mathrm{~h}$ & Within $4 \mathrm{~h}$ & Contralateral & $\begin{array}{l}\text { Yes (4) } \\
\text { No (1) }\end{array}$ & 5-7 days & Good \\
\hline Shimizu et al., 2009²) & $56 / \mathrm{F}$ & Tentorial meningioma & Park-bench & $10 \mathrm{~h}$ & $2 \mathrm{~h}$ & Contralateral & Yes & 11 days & $\begin{array}{l}\text { Brachial } \\
\text { plexopathy }\end{array}$ \\
\hline $\begin{array}{l}\text { Hébert-Blouin et al., } \\
2009^{3)}\end{array}$ & $53 / \mathrm{F}$ & Frontal metastatic lesion & Supine & ND & Immediate & Contralateral & ND & ND & $\begin{array}{c}\text { Brachial } \\
\text { plexopathy }\end{array}$ \\
\hline Cavaliere et al., 20096) & $73 / \mathrm{F}$ & $\begin{array}{l}\text { Parieto-occipital } \\
\text { meningioma }\end{array}$ & Prone & $7 \mathrm{~h}$ & $1 \mathrm{~h}$ & Contralateral & Yes & 7 days & Good \\
\hline Singha, $2009^{1)}$ & $23 / \mathrm{F}$ & Cerebellar meningioma & Lateral & ND & Immediate & Contralateral & Yes & 7 days & Good \\
\hline Prabhu et al., $2010^{7)}$ & $41 / \mathrm{M}$ & VS & Semi-sitting & ND & $4 \mathrm{~h}$ & Contralateral & Yes & 7 days & Good \\
\hline Diehn et al., 2012 & $51 / \mathrm{F}$ & $\begin{array}{c}\text { Glossopharyngeal } \\
\text { neuralgia }\end{array}$ & ND & $2 \mathrm{~h} 40 \mathrm{~m}$ & Immediate & Contralateral & Yes & 2 days & Good \\
\hline \multirow[t]{2}{*}{ Present cases } & 20/M & VS & Park-bench & $8 \mathrm{~h}$ & $4 \mathrm{~h}$ & Contralateral & Yes & 4 days & Good \\
\hline & $71 / \mathrm{F}$ & Hemifacial spasm & Lateral & $5 \mathrm{~h}$ & $2 \mathrm{~h}$ & Contralateral & Yes & 7 days & Good \\
\hline
\end{tabular}

F: female, M: male, ND: not described, VS: vestibular schwannoma.

the side opposite to the surgical procedure. These facts strongly indicate that head rotation and flexion during surgery could be related to the cause of this complication. The submandibular duct opens on the sublingual papilla and it accompanies the lingual nerve in the base of the tongue (Fig. 3). ${ }^{9,10)}$ The tongue numbness observed in Case 1 suggested the compression of the submandibular duct.9) $\mathrm{Com}$ pression of the tongue by the endotracheal tube could potentially cause salivary duct obstruction. Mechanical obstruction of the duct could lead to salivary stasis and secondary bacterial infection. Finally, these conditions could cause obstructive sialadenitis (Fig. 4).

Furthermore, some authors have pointed out that the pressure ischemia of the submandibular gland (ischemic sialadenitis) and surrounding soft tissue might also result in this rare complication. ${ }^{1,6,11)}$ Compression of these tissues by the endotracheal tube may cause their temporary ischemia. Head rotation may also cause a compression of the facial artery itself, which supplies the submandibular gland and could lead to ischemia. The compressions are released when the head position is returned after the surgery, at which point reperfusion injury of these tissues may occur. Reperfusion injury following ischemia of these tissues could explain the clinical features of the neck swelling that develops a few hours after surgery (Table 1). The intrinsic vascular network of the submandibular gland and surrounding tissues might be more related to the occurrence of the ischemic sialadenitis than patients' physique or age, according to the present cases and the literature.

Although precise pathogenesis of this rare surgical complication remains to be clarified, either or both of the mechanisms described above would contribute to its occurrence.

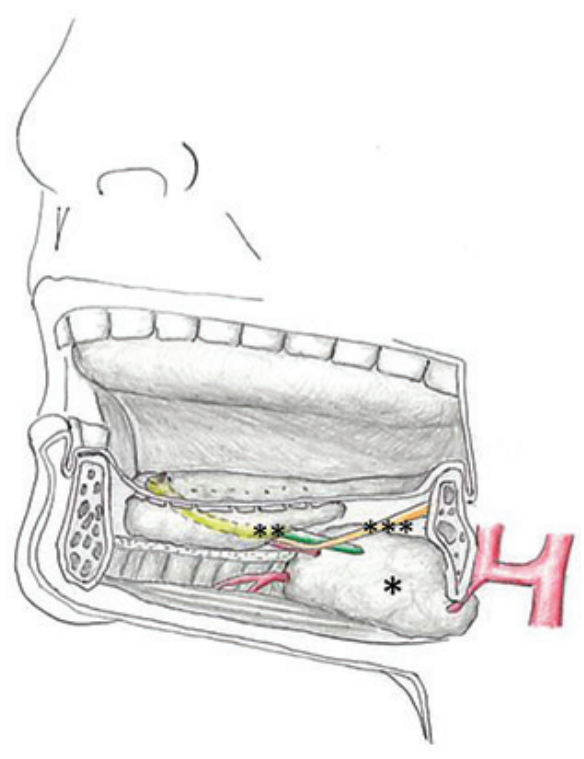

Fig. 4 Schema of the submandibular gland (*) and surrounding tissues. The body of the mandible is partly removed. Submandibular duct $(* *)$ runs parallel to lingual nerve $(* * *)$ under either side of the tongue. The facial artery (arrow) arises from the external carotid artery and runs a tortuous course extending obliquely upward over the body of the mandible. Its four or five terminal branches supply the submandibular gland around the lower border of the mandible.

We placed the endotracheal tube on the contralateral side of surgery to make room for facial EMG response monitoring in the present cases. This might enhance the compression of the submandibular gland and the surrounding tissues. Furthermore, the size of the patients' tongue and its swelling 
during surgery may also be involved in compression, as the patients' tongue will shift downward (contralateral to the surgical site) due to gravity. The position and the size of bite blocks used during surgery may be similarly related. Therefore, it is recommended that anesthesiologists secure the endotracheal tube on the ipsilateral side of the surgery, to reduce tissue compression. No acute submandibular sialadenitis has been observed thereafter, although further investigation would be needed to confirm it. While patients' age, sex, and physique or duration of surgeries may not be predictors of postoperative submandibular sialadenitis, it is reported to occur more often following posterior fossa surgery. This rare complication should be considered with careful observation, particularly following surgeries in the posterior fossa region. When it occurs, early airway protection should be prioritized, and the following conservative treatment could provide a good outcome.

\section{Conflicts of Interest Disclosure}

The authors have no financial interest or ethical conflicts to disclose.

\section{References}

1) Singha SK, Chatterjee N: Postoperative sialadenitis following retromastoid suboccipital craniectomy for posterior fossa tumor. $J$ Anesth 23: 591-593, 2009
2) Shimizu S, Sato K, Mabuchi I, Utsuki S, Oka H, Kan S, Fujii K: Brachial plexopathy due to massive swelling of the neck associated with craniotomy in the park bench position. Surg Neurol 71: 504-508; discussion 508-509, 2009

3) Hébert-Blouin MN, Chowdhry SA, Abrahams PH, Spinner RJ: An unusual anatomical explanation for contralateral upper extremity weakness after frontal craniotomy. Clin Anat 22: 840-845, 2009

4) Kim LJ, Klopfenstein JD, Feiz-Erfan I, Zubay GP, Spetzler RF: Postoperative acute sialadenitis after skull base surgery. Skull Base 18: 129-134, 2008

5) Izci Y, Erdogan E, Timurkaynak E: Acute right submandibular swelling following surgery for bilateral optic nerve meningioma. J Neurosurg Anesthesiol 17: 58-59, 2005

6) Cavaliere F, Conti G, Annetta MG, Greco A, Cina A, Proietti R: Massive facial edema and airway obstruction secondary to acute postoperative sialadenitis or "anesthesia mumps": a case report. J Med Case Rep 3: 7073, 2009

7) Prabhu K, Ramamani, Nair S, Chacko AG: Acute submandibular sialadenitis as a cause of unilateral neck swelling after posterior fossa surgery in sitting position. Neurol India 58: 963-964, 2010

8) Diehn FE, Morris JM: Acute contralateral submandibular sialadenitis as a complication of skull base surgery. Clin Neuroradiol 22: 161-163, 2012

9) Foley E, Mc Dermott TE, Shanahan E, Phelan D: Transient isolated lingual nerve neuropraxia associated with general anaesthesia and laryngeal mask use: two case reports and a review of the literature. Ir $J$ Med Sci 179: 297-300, 2010

10) Michael Schuenke, Erik Schulte, Udo Schumacher, Lawrence M. Ross, Edward D. Lamperti, Ethan Taub, Markus Voll, Karl Wesker: Thieme Atlas of Anatomy: Head and Neuroanatomy. New York, Thieme, 2010

11) Fujisawa Y, Aiyama S: Histochemical and chronological analysis of mouse submandibular gland parenchyma subjected to abrupt reperfusion. Histol Histopathol 18: 871-878, 2003

Corresponding author:

Shunsuke Terasaka, MD, Department of Neurosurgery, Graduate School of Medicine, Hokkaido University, North 15 West 7, Kita-ku, Sapporo, Hokkaido 060-8638, Japan. 